\title{
A New Mixed Formulation for Elasticity
}

\author{
Douglas N. Arnold ${ }^{1, \star}$ and Richard S. Falk ${ }^{2, \star \star}$ \\ ${ }^{1}$ Department of Mathematics, University of Maryland, College Park, MD 20742, USA \\ ${ }^{2}$ Department of Mathematics, Rutgers University, New Brunswick, NJ 08903, USA
}

Dedicated to Professor Ivo Babuška on the occasion of his sixtieth birthday

\begin{abstract}
Summary. We propose a new mixed variational formulation for the equations of linear elasticity. It does not require symmetric tensors and consequently is easy to discretize by adapting mixed finite elements developed for scalar second order elliptic equations.
\end{abstract}

Subject Classifications: AMS(MOS): 65N30; 73C35; 73K25; CR: G1.8.

\section{Introduction}

In this paper we present a new mixed variational formulation for the problem of linear elastostatics. Our formulation is very similar to the classical HellingerReissner formulation, but appears superior for finite element discretization. To make plain the relation between the Hellinger-Reissner formulation and the present one, we consider first an elastic body occupying a region $\Omega$ in Euclidean $n$-space ( $n=2$ or 3 ) subject to given body forces $f$ and whose displacement $\mathrm{g}$ on $\Gamma=\partial \Omega$ is known. The Hellinger-Reissner principle seeks a saddle-point of the quadratic functional

$$
\mathscr{I}(\underset{\approx}{\tau}, \underset{\approx}{v})=\int_{\Omega}\left[\frac{1}{2} \mathbf{A} \underset{\approx}{\tau}: \underset{\tau}{\tau}+\operatorname{div} \underset{\sim}{\tau} \cdot \underset{\sim}{v}-f \cdot \underset{\sim}{v}\right]-\int_{\partial \Omega} g \cdot \underset{\approx}{\tau} n .
$$

The variables $\underset{\sim}{\tau}$ and $\underset{\sim}{v}$ range over spaces of suitably smooth functions on $\Omega$ with values in $\underset{\widetilde{R}}{\mathbb{R}}$, the space of symmetric $n \times n$ tensors, and $\mathbb{R}=\mathbb{R}^{n}$, respectively. The fourth order tensor $\mathbf{A}$ is the compliance tensor, which characterizes the elastic properities of the material. Further notations are explained in Sect. 2. Under reasonable assumptions there is a unique saddle-point $(\underset{\approx}{g}, \underset{\sim}{u})$ of $(1.1)$ and, moreover, $\underset{Z}{ }$ is the stress field and $\underline{u}$ the displacement field. The Euler-Lagrange equations associated with (1.1) form an elliptic system of order $2 n$.

The present formulation also seeks a saddle-point of a quadratic functional of the form (1.1). The functional differs only in that the compliance tensor is

\footnotetext{
* Supported by NSF Grant DMS-8313247

* Supported by NSF Grant DMS-8402616
} 
replaced by a different fourth order tensor, which depends on $\mathbf{A}$ in a simple fashion. A more essential difference is that in our formulation the variable $\tau$ ranges over all suitably smooth functions with values in $\underset{\tilde{z}}{\mathbb{R}}$ (all $n \times n$ tensors) rather than $\underset{\approx}{\mathbb{R}}$ (symmetric tensors). Again there is a unique saddle-point, $(\underline{\underline{\approx}}, \underline{u})$. The vector-valued component $\underline{u}$ is again the displacement field, but the tensorvalued component $\rho$ does not coincide with the stress field $\sigma$. This "pseudostress" field contains more information than $\underset{\approx}{\sigma}$ in that $\underset{\approx}{\sigma}$ may $\tilde{\tilde{b}}$ determined from $\underset{\approx}{\rho}$ simply as a linear combination of its components, but $\sigma$ does not determine $\underset{\sigma}{\rho}$. In fact from $\underset{\approx}{\rho}$ one may determine the gradient of the displacement while $\underset{\tilde{\sigma}}{\sigma}$ only determines its symmetric part.

The construction of effective finite elements for the Hellinger-Reissner principle has proven to be very difficult and has not yet been accomplished in a completely satisfactory manner for plane elasticity problems (see [3] for a discussion on this point). For three dimensional problems no useful stable elements are known. For the simpler problem of the discretization of a mixed formulation of second order elliptic problems, a number of excellent finite elements have been devised and thoroughly studied, but these elements cannot be simply adapted to the Hellinger-Reissner formulation in order to solve the elasticity problem. The crux of the difficulty is the requirement that the tensors occurring in the Hellinger-Reissner formulation be symmetric. Our formulation eliminates this requirement and so enables the direct adaptation of these elements to the elasticity problem.

Another alternative to the Hellinger-Reissner variational formulation has been presented by Arnold et al. [2] for the case of plane elasticity. They also sought a mixed variational formulation of elasticity which avoided the necessity of constructing symmetric tensor elements for discretization. However the formulation they proposed is quite different from the one presented here. They retained the basic stress and displacement variables, $\sigma$ and $\underset{\sim}{u}$, but instead of seeking a saddle-point of the functional $\mathscr{I}(\tau, v)$ in $(1 . \tilde{\tilde{1}})$ with the tensor $\tau$ constrained to be symmetric (the usual Hellinger-Reissner formulation), they added a Lagrange multiplier to the functional and then sought a saddle-point without a symmetry constraint on $\tau$. The effect of the Lagrange multiplier is to enforce the symmetry of the tensor variable. Thus in their formulation there are three variables: the stress, the displacement, and the Lagrange multiplier, which is scalar-valued and turns out to coincide with the rotation of the displacement. To discretize one must construct finite element spaces for each of these variables satisfying appropriate conditions. In contrast to the present formulation, it is not obvious how to do this even in light of good elements for the second order problem. In [2], Arnold et al. constructed one such family of spaces and proved stability and convergence.

An outline of the paper is as follows. In the next section we collect the notation to be used in the remainder of the paper and in Sect. 3 derive the new variational principle. Because the stress is not a fundamental unknown in this formulation, it is not obvious how to modify it in case the traction rather than the displacement is given on part of the boundary. In Sect. 4 we present such a modification and discuss its discretization. An analysis of a particular method of discretization is then given in Sect. 5. Finally in Sect. 6 we discuss the application of our formulation to incompressible elasticity. 


\section{Notations and Preliminaries}

We shall consider the system of elasticity on a smoothly bounded domain $\Omega \subset \mathbb{R}=\mathbb{R}^{n}, n=2$ or 3 . We use the following notational conventions. Lower case letters and names of spaces are underscored by $\sim$ and $\approx$ to denote $n$-vectors and $n \times n$ tensors respectively. Fourth order tensors are denoted by boldface capital letters. The components of vectors and tensors are denoted by the corresponding subscripted lower case letters. The product of a fourth order tensor and a second order one is second order; thus $\underset{\sim}{\tau}=\mathbf{A} \underset{\sim}{\sigma}$ means

$$
\tau_{i j}=\sum_{k, l=1}^{n} a_{i j k l} \sigma_{k l}, \quad 1 \leqq i, j \leqq n
$$

We shall also use the notations

$$
\underset{\approx}{g}: \tau=\sum_{k, l=1}^{n} \sigma_{k l} \tau_{k l} \quad \text { and } \quad|\underset{\approx}{\tau}|=(\underset{\approx}{\tau}: \tau)^{1 / 2}
$$

If $X$ is a space of scalars (or scalar-valued functions), we use $X$ to denote the space of vectors with components in $X$. If $Y$ is a space of vectors, $\underset{\sim}{Y}$ denotes the space of tensors with rows in $Y$ and $\underset{z}{Y_{s}}$ the subspace consisting of symmetric tensors.

In addition to the usual Sobolev spaces $H^{s}(\Omega)$ and $H^{s}(\Gamma)$, we will use the space $H(\operatorname{div}, \Omega$ ) of square integrable functions on $\Omega$ with square integrable diver-

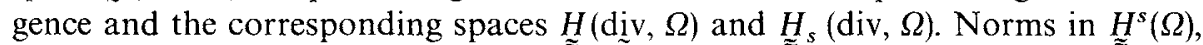
$H^{s}(\Omega)$, and $H^{s}(\Omega)$ will be denoted by $\|\cdot\|_{s}$ (the choice will be clear from the context) and norms in $H^{s}\left(\Gamma_{i}\right)$ and $H^{s}\left(\Gamma_{i}\right)\left(\Gamma_{i}\right.$ a subset of $\left.\Gamma=\partial \Omega\right)$ by $|\cdot|_{s . r_{i}}$.

A linearly elastic material (possibly anisotropic and inhomogeneous) is characterized by its compliance tensor $\mathbf{A}=\mathbf{A}(\underset{\sim}{x})$ whose components are functions in $\Omega$ satisfying

$$
a_{i j k l}=a_{k l i j}=a_{j i k l}, \quad 1 \leqq i, j, k, l \leqq n .
$$

This condition means that for each $x \in \Omega, \mathbf{A}(x)$ is a self-adjoint linear operator on $\underset{\approx}{\mathbb{R}}$ which annihilates antisymmetric tensors and maps $\underset{\approx}{\mathbb{R}}$ into itself. We assume (except in Sect. 6) that the restriction of $\mathbf{A}(\underset{\tilde{x})}{\boldsymbol{x}}$ to $\underset{\tilde{z}}{\mathbb{R}}$ is positive definite and bounded uniformly in $\underset{\sim}{x}$, so that there exist positive constants $\gamma_{0}$ and $c_{0}$ with

$$
\gamma_{0}|\underset{\sim}{\tau}|^{2} \leqq \underset{\approx}{\tau}: \mathbf{A}(x) \underset{\approx}{\tau} \leqq c_{0}|\underset{\approx}{\tau}|^{2}, \quad \underset{\approx}{\tau} \underset{\approx}{\mathbb{R}}, \quad \underset{\sim}{x} \in \Omega
$$

Consequently we may define the elasticity tensor $\mathbf{C}(x)$ by the equations

and

$$
\underset{\approx}{\tau}=\mathbf{A C} \underset{\approx}{\tau}=\mathbf{C A} \underset{\approx}{\tau}, \quad \underset{\approx}{\tau} \in \mathbb{R}_{\Sigma},
$$

Clearly

$$
c_{i j k l}=c_{k l i j}=c_{j i k l}, \quad 1 \leqq i, j, k, l \leqq n .
$$

$$
\gamma_{1}|\underset{\approx}{\tau}|^{2} \leqq \underset{\approx}{ }: \mathbf{C}(x) \underset{\approx}{\tau} \leqq c_{1}|\underset{\tau}{\tau}|^{2}, \quad \underset{\approx}{\tau} \in \underset{\approx}{\mathbb{R}}, \quad x \in \Omega,
$$

with $\gamma_{1}=c_{0}^{-1}, c_{1}=\gamma_{0}^{-1}$.

We shall be considering in the following sections the equations of elasticity 


$$
\begin{aligned}
\mathbf{A} \underset{\approx}{\sigma}=\underset{\approx}{\xi(u)} & \text { in } \Omega, \\
\operatorname{div}_{\sim}^{\sigma}=\underset{\sim}{f} & \text { in } \Omega,
\end{aligned}
$$

where $\underset{\approx}{\varepsilon}(\underset{\sim}{u})=\left[\operatorname{grad} \underset{\sim}{u}+(\operatorname{grad} \underset{\approx}{t})^{t}\right] / 2$ and $\operatorname{grad} \underset{\approx}{u}$ is the Jacobian matrix, subject to the boundary conditions

$$
\begin{array}{cc}
\stackrel{u}{u}=g_{1} & \text { on } \Gamma_{1}, \\
\underset{\sim}{\sim} \underset{\sim}{n}=g_{2} & \text { on } \Gamma_{2},
\end{array}
$$

where $\Gamma_{1}$ and $\Gamma_{2}$ are disjoint closed subsets of $\Gamma$ with $\Gamma_{1} \cup \Gamma_{2}=\Gamma$. In Sect. 3, $\Gamma_{2}$ will be empty and in Sects. 4 and 5 both $\Gamma_{1}$ and $\Gamma_{2}$ nonempty.

\section{The New Mixed Formulation}

The Euler-Lagrange equations associated with the Hellinger-Reissner principle (cf. (1.1)) are

$$
\begin{aligned}
\mathbf{A} \underset{\approx}{\sigma}=\underset{\sim}{g}(u) & \text { in } \Omega, \\
\operatorname{div} \underset{\approx}{\sigma}=\underset{\sim}{f} & \text { in } \Omega, \\
\underset{\sim}{u} & \text { on } \Gamma .
\end{aligned}
$$

The constitutive Eqs. (3.1) can also be stated in terms of the elasticity tensor and displacement gradient

$$
\underset{\approx}{\sigma}=\mathbf{C} \underset{\approx}{\operatorname{grad}} \underset{\sim}{u}
$$

(by (2.2) $\mathbf{C g r a d}_{\approx} \underset{\approx}{u}=\mathbf{C} \underset{\approx}{g}(u)$ ). We define the pseudostress $\underset{\approx}{\rho}$ by the equation

$$
\underset{\approx}{\rho}=(\mathbf{C}+\beta \mathbf{D}) \underset{\approx}{\operatorname{grad}} \underline{\sim}
$$

where $\beta$ is a positive constant and

$$
\mathbf{D} \underset{\approx}{\tau}=\operatorname{tr}(\tau) \underset{\approx}{\delta}-\tau_{\approx}^{t}
$$

(In the above, $\operatorname{tr}(\tau)$ is the trace of $\underset{\approx}{\tau}, \tau_{\approx}^{t}$ the transpose of $\underset{\approx}{\tau}$, and $\delta$ the $3 \times 3$ identity matrix.) From the calculus identity

$$
\operatorname{div}[(\operatorname{div} \underset{\sim}{u}) \underset{\approx}{\delta}]=\operatorname{grad}(\operatorname{div} \underset{\sim}{u})=\operatorname{div}\left(\operatorname{grad} \underset{\sim}{\mathfrak{u}^{t}}\right)
$$

and the equilibrium Eq. (3.2) it follows that

$$
\operatorname{div} \underset{\sim}{\rho}=f .
$$

We shall now show that if $\beta$ is chosen appropriately, $\mathbf{C}+\beta \mathbf{D}$ defines an invertible operator on $\underset{\approx}{\mathbb{R}}$ (for each $\underset{\approx}{x \in} \Omega$ ) and hence (3.5) may be inverted to give

$$
\mathbf{B} \underset{\approx}{\rho}=\operatorname{grad} \underset{\approx}{u} .
$$

In contrast, $\mathbf{C}$ does not define an invertible operator on $\underset{\approx}{\mathbb{R}}$ and (3.4) may not be inverted in this way. 
There is some freedom in the choice of $\beta$. Taking $\beta=\gamma_{1} / 2$ where $\gamma_{1}$ is any constant for which (2.3) holds, we now show

$$
(\mathbf{C}+\beta \mathbf{D}) \underset{\approx}{\tau}: \tau \geqq \gamma_{1}|\underset{\approx}{\tau}|^{2} / 2, \quad \underset{\approx}{\tau} \in \mathbb{\approx} .
$$

Indeed by (2.2) and (2.3)

$$
\mathbf{C} \underset{\approx}{\tau}: \tau=\mathbf{C}[(\underset{\approx}{\tau}+\underset{\approx}{\tau}) / 2]:[(\underset{\approx}{\tau}+\underset{\approx}{\tau}) / 2] \geqq \gamma_{1}|(\underset{\approx}{\tau}+\underset{\approx}{\tau}) / 2|^{2}=\gamma_{1}\left(|\underset{\approx}{\tau}|^{2}+\underset{\approx}{\tau}: \tau^{t}\right) / 2,
$$

and by (3.6)

$$
\mathbf{D} \underset{\approx}{\tau}: \underset{\tau}{\tau}=\operatorname{tr}(\underset{\sim}{\tau})^{2}-\underset{\approx}{\tau}: \underset{\tau}{\tau} \geqq-\underset{\approx}{\tau}: \underset{\tau}{\tau}
$$

Thus $\mathbf{C}(x)+\beta \mathbf{D}$ is positive definite on $\underset{\approx}{\mathbb{R}}$ (not just $\mathbb{Z}_{s}$ ), and denoting by $\mathbf{B}(x)$ its inverse we have (3.8). Moreover since $|\mathbf{D} \underset{\approx}{\tau}| \leqq 2|\tau| \tau \mid$ for all $\tau \in \mathbb{R}$, we also have

Thus

$$
(\mathbf{C}+\beta \mathbf{D}) \underset{\approx}{\tau}: \tau \leqq\left(c_{1}+\gamma_{1}\right)|\underset{\tau}{\tau}|^{2} .
$$

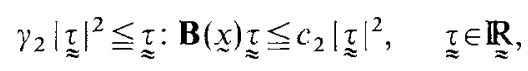

with $\gamma_{2}=\left(c_{1}+\gamma_{1}\right)^{-1}, c_{2}=2 / \gamma_{1}$.

To summarize the foregoing considerations, we propose to discretize the boundary value problem (3.8), (3.7), (3.3) rather than the classical problem (3.1)-(3.3). Alternately we propose in place of the classical Hellinger-Reissner principle to seek a saddle-point $(\underset{\sim}{\rho, u})$ in $\underset{\approx}{H}(\operatorname{div}, \Omega) \times{\underset{\sim}{2}}^{2}(\Omega)$ of the quadratic functional

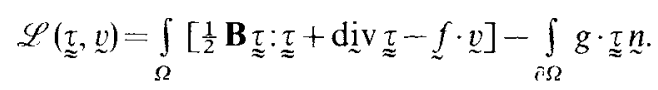

Existence and uniqueness of the saddle-point follow from the theorem of Brezzi [9] once we verify the conditions

$$
\begin{aligned}
& \int_{\Omega} \mathbf{B} \underset{\tau}{\approx}: \tau \geqq \gamma\|\tau\|_{0}^{2}, \quad \underset{\approx}{\approx} \underset{\sim}{\stackrel{L^{2}}{\approx}(\Omega),}
\end{aligned}
$$

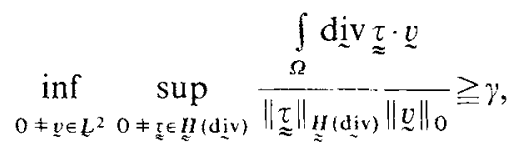

for some $\gamma>0$. The first condition follows immediately from (3.9). The second follows from the equality $\operatorname{div} \underset{\sim}{H}(\operatorname{div}, \Omega)=L_{\sim}^{2}(\Omega)$, itself an obvious consequence of the equality $\operatorname{div} \underset{\sim}{H}(\operatorname{div}, \Omega)=\tilde{L}^{2}(\Omega)$. Thus the pseudostress and displacement are the unique solution of the saddle-point problem associated with $\mathscr{L}$ or of the Euler-Lagrange equations (3.8), (3.7), (3.3). In light of (3.4) and (3.8), the stress $g$ may be recovered (without differentiating) from the relation

$$
\underset{\approx}{\sigma}=\mathbf{C B} \underset{\approx}{\rho}=\underset{\approx}{\rho}-\beta \mathbf{D B} \rho
$$

A mixed finite element for our formulation defines an approximate solution $\left(\rho_{h}, u_{h}\right)$ as the saddle-point of $\mathscr{L}$ restricted to a finite-dimensional space $\underline{S}_{h} \times V_{h}$

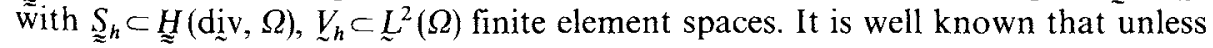


the subspaces ${\underset{\approx}{S_{h}}}_{h}$ and $V_{h}$ are chosen appropriately, the restriction of $\mathscr{L}$ need not have a unique saddle-point and even if it does, good approximability of $(\rho, u)$ by $S_{h} \times V_{h}$ need not insure accurate approximation by $\left(\rho_{h}, u_{h}\right)$. Various stability conditions have been proposed which, togethẽ with good approximability by ${\underset{\Sigma}{h}}_{h}$ and $V_{h}$, insure the existence and uniqueness of $\left(\rho_{h}, u_{h}\right)$

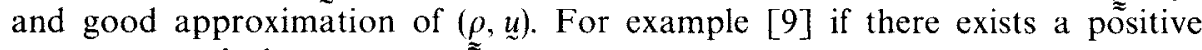
constant $\gamma$ such that

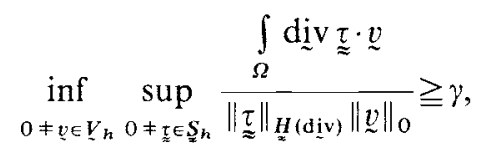

one obtains existence, uniqueness, and the quasioptimal estimate

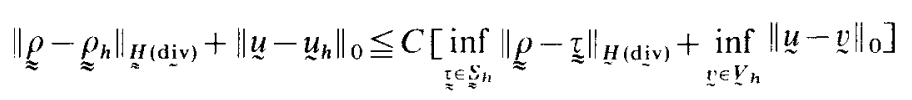

with $C$ depending on the stability constant $\gamma$. Scalar analogues of the following additional stability properties have also been used by a number of authors:

There exists a projection operator $\Pi_{h}: \underset{\approx}{H^{1}} \rightarrow \underset{\approx}{S_{h}}$ having the property

$$
\begin{gathered}
\int_{\Omega} \operatorname{div}\left(\underset{\sim}{\tau}-\Pi_{h} \underset{\approx}{\tau}\right) \cdot \underset{\sim}{v}=0, \quad \underset{\sim}{v} \in V_{h}, \\
\operatorname{div} \underset{\approx}{S_{h}} \subset V_{h} .
\end{gathered}
$$

Using these hypotheses and usual approximation properties, a variety of estimates including $L^{2}$ estimates, negative norm estimates, interior estimates, $L^{\infty}$ estimates, etc., may be established by basically standard techniques (cf. [3,12-15, 19, 22]).

For our formulation (in contrast to the Hellinger-Reissner formulation) it is straightforward to construct finite element spaces $\underset{\approx}{S_{h}}$ and $V_{h}$ satisfying all the stability conditions (3.13), (3.15), and (3.16). To see this we recall the solution by mixed finite element methods of a second order elliptic problem. Here we seek to approximate the unique saddle-point $(\rho, u)$ in $\underset{\sim}{H}(\operatorname{div}, \Omega) \times L^{2}(\Omega)$ of the functional

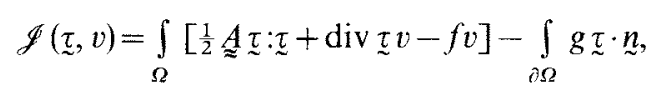

where $f \in L^{2}(\Omega), g \in H^{1 / 2}(\partial \Omega)$, and the $n \times n$ matrix $\underset{\approx}{A}$ are given. An approximation is found by selecting finite dimensional subspaces $S_{h} \subset H\left(\right.$ div, $\Omega$ ), $V_{h} \subset L^{2}(\Omega)$ and determining the approximate solution $\left(\varrho_{h}, u_{h}\right)$ as the unique saddle-point of $\mathscr{J}$ restricted to $S_{h} \times V_{h}$ (if this exists). Several families of finite element spaces have been constructed which satisfy the analogues of the stability conditions (3.13). (3.15), (3.16), afford good approximability, and can be efficiently implemented. The best known of these are the spaces of Raviart and Thomas [19,22], and Nedelec [16]. In the case of a two dimensional domain, Raviart and Thomas constructed for each triangulation $\mathscr{T}_{h}$ and each nonnegative order $k$, spaces $S_{h}$ and $V_{h}$ and verified the analogue of the stability condition (3.13) with $\gamma$ independent of the triangulation (depending only on a lower bound for the minimal angle). They also derived quadrilateral elements. Nedelec [16] simplified 
their elements and extended them to three dimensions. The Raviart-ThomasNedelec elements have been thoroughly analyzed both from the point of view of accuracy and implementation $[1,11-15,20,21]$. For the two dimensional case, a second family of spaces satisfying the Brezzi conditions has been recently introduced by Brezzi et al. [10]. Their spaces are also defined for arbitrary triangulations (and for quadrilateral subdivisions) and arbitrary order. In all, one may say that excellent mixed finite element methods for second order elliptic problems are known and well understood.

Returning to our formulation of the elasticity problem, it is easy to see that conditions (3.13), (3.15), and (3.16) are satisfied if we take

$$
S_{h}=S_{h} \times S_{h}, \quad V_{h}=V_{h} \times V_{h},
$$

where $S_{h} \subset H($ div,$\Omega), V_{h} \subset L^{2}(\Omega)$, satisfy the analogous conditions for discretization of the mixed second order problem. We may use, for example, the RaviartThomas-Nedelec or Brezzi-Douglas-Marini elements with our formulation.

\section{Traction Boundary Conditions}

In this section we show how to modify our formulation in case the displacement boundary condition is replaced by a traction boundary condition on part of the boundary. Thus the boundary conditions we consider are

$$
\begin{array}{cc}
\underset{u}{u}=\underset{\approx}{g_{1}} & \text { on } \Gamma_{1}, \\
\underset{\sim}{\boldsymbol{n}}=\underset{\approx}{g_{2}} & \text { on } \Gamma_{2},
\end{array}
$$

where $\Gamma_{1}$ and $\Gamma_{2}$ are disjoint nonempty closed subsets of $\Gamma$ with $\Gamma_{1} \cup \Gamma_{2}=\Gamma$. Note that we have assumed that the displacement and traction boundary conditions are given on separated boundary curves or surfaces. This (rather strong) assumption is made to avoid various technicalities. Since the stress $\underline{\sigma}$ has been eliminated from our formulation, it is not obvious how to include the condition (4.1). In order to do this we first define the tangential gradient of $\underset{\sim}{u}$ by $\operatorname{grad}_{\Gamma} \underline{\sim}$

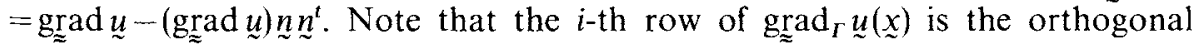
projection of grad $\tilde{u}_{i}(x)$ onto the tangent space of $\Gamma$ at $\underset{\sim}{x}$. Consequently, $\operatorname{grad}_{\tilde{\tau}} \underline{u}$ depends only on $\left.\underset{\sim}{u}\right|_{\Gamma}$, so $\operatorname{grad}_{\Gamma}$ may be viewed as a bounded map of ${\underset{\sim}{H}}^{\tilde{1} / 2}\left(\Gamma_{2}\right)$ into $H_{\sim}^{-1 / 2}\left(\Gamma_{2}\right)$. For $\mu \in H^{1 / 2}\left(\Gamma_{2}\right)$ define

$$
b(\underset{\sim}{\mu})=-\beta \mathbf{D}\left(\operatorname{grad}_{\approx} \underset{\sim}{\mu}\right) \underset{\sim}{n} .
$$

For $\underset{\sim}{u \in H_{\sim}}{ }^{1}(\Omega)$ we write $\underset{\sim}{b}(\underset{\sim}{u})$ in place of $\underset{\sim}{b}\left(\tau_{\Gamma_{2}}\right)$.

Lemma 4.1. The operator $\underset{\sim}{b}: \underset{\sim}{H^{1 / 2}}\left(\Gamma_{2}\right) \rightarrow \underset{\sim}{H^{-1 / 2}}\left(\Gamma_{2}\right)$ is bounded and self-adjoint. Moreover, for $\underset{\sim}{u \in H_{\sim}}{ }^{1}(\Omega)$,

$$
\underline{b}(\underline{u})=-\beta \mathbf{D}(\underset{\sim}{\operatorname{grad}} \underset{\sim}{u}) \underset{\sim}{n} .
$$

Proof. The second statement follows from the identity

$$
\mathbf{D}\left(\underset{\sim}{\tau} \underset{\sim}{n} \underline{\sim}^{t}\right)=\operatorname{tr}\left(\underset{\sim}{\tau} \underline{\sim} \underline{n}^{t}\right) \underline{\sim}-\left(\underline{n}^{t} \underset{\sim}{\tau} \underline{\sim}\right) \underline{\sim}=0,
$$


which holds for any $\underset{\approx}{\tau} \underset{\approx}{\mathbb{R}}$. To prove the first statement, we define $(\underset{\approx}{\sigma} \underset{\sim}{u}, \underset{\approx}{\rho})$ and

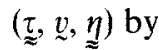

$$
\begin{aligned}
& \mathbf{A} \underset{\approx}{\sigma}-\underset{\approx}{\varepsilon}(u)=0, \quad \mathbf{A} \underset{\approx}{\tau}-\underset{\approx}{\varepsilon}(v)=0, \\
& \operatorname{div} \underset{\sim}{g}=0, \quad \operatorname{div} \underset{\sim}{\tau}=0, \\
& \underset{\sim}{u}=0 \quad \text { on } \Gamma_{1}, \quad \underset{v}{v}=0 \quad \text { on } \Gamma_{1}, \\
& \underset{\sim}{u}=\mu \quad \text { on } \Gamma_{2}, \quad \underline{v}=\underset{\sim}{\lambda} \quad \text { on } \Gamma_{2}, \\
& \stackrel{\rho}{\rho}=\underset{\approx}{\sigma}+\beta \mathbf{D}\left(\operatorname{grad}_{\approx}^{u}\right), \quad \underline{\approx}=\underset{\approx}{\tau}+\beta \mathbf{D}(\operatorname{grad} v) .
\end{aligned}
$$

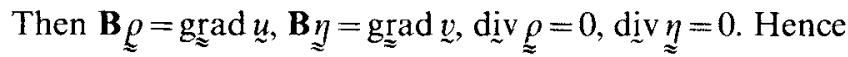

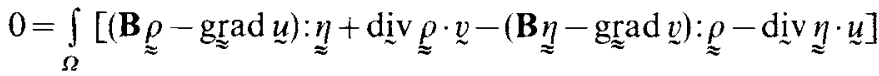

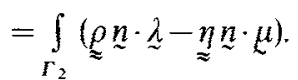

Also

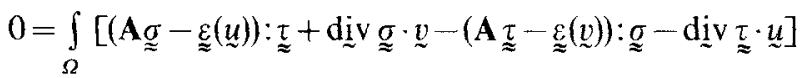

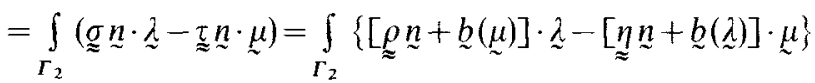

$$
\begin{aligned}
& =\int_{\Gamma_{2}}[\underline{b}(\underline{\mu}) \cdot \underset{\sim}{\lambda}-\underset{\sim}{b}(\underset{\sim}{\lambda}) \cdot \underset{\sim}{\mu}] .
\end{aligned}
$$

This establishes the self-adjointness.

It follows from (4.2) that the boundary condition (4.1) may be restated as

$$
\underset{\sim}{\rho} \underset{\sim}{n}+\underset{\sim}{b}(u)=g_{2} \quad \text { on } \Gamma_{2} \text {. }
$$

For the traction problem we seek a critical point $\underset{\sim}{\rho}, \underset{\sim}{u}, \underset{\sim}{\lambda}) \in \underset{\sim}{H}(\operatorname{div}, \Omega) \times \underset{\sim}{L^{2}}(\Omega)$ $\times{\underset{\sim}{H}}^{1 / 2}\left(\Gamma_{2}\right)$ of the functional

$$
\begin{aligned}
& \overline{\mathscr{L}}(\underline{\tau}, \underset{\sim}{\mu} \underset{\sim}{\mu})=\int_{\Omega}\left[\frac{1}{2} \mathbf{B} \underset{\sim}{\tau}: \underset{\tau}{\tau}+\operatorname{div} \underset{\sim}{\tau} \cdot v-f \cdot \underset{v}{f}\right] \\
& -\int_{\Gamma_{1}}{\underset{g}{1}}_{1} \cdot \underset{\approx}{\tau} \underset{\sim}{n}-\int_{\Gamma_{2}}\left(\frac{1}{2} \underset{\sim}{b}(\mu)+\underset{\sim}{\tau} \underset{\sim}{\sim}-g_{2}\right) \cdot \underset{\sim}{\mu} .
\end{aligned}
$$

A weak form of this problem is:

Find $(\underset{\sim}{\rho}, \underset{\sim}{u}, \underset{\sim}{\lambda}) \in \underset{\sim}{H}(\operatorname{div}, \Omega) \times{\underset{\sim}{L}}^{2}(\Omega) \times \underline{\sim}^{1 / 2}\left(\Gamma_{2}\right)$ such that

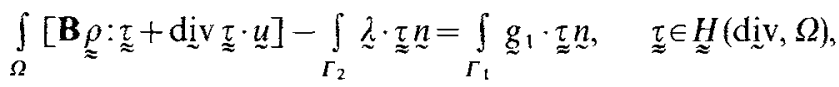

$$
\begin{aligned}
& \int_{\Omega} \operatorname{div} \underset{\sim}{\rho} \cdot \underset{\sim}{v}=\int_{\Omega} \underset{\sim}{f} \cdot \underset{\sim}{v}, \quad v \in L_{\sim}^{2}(\Omega), \\
& \int_{\Gamma_{2}}[\underset{\sim}{\rho} \underset{\sim}{n}+\underset{\sim}{b}(\lambda)] \cdot \underset{\sim}{\mu}=\int_{\Gamma_{2}}{\underset{\sim}{2}}_{2} \cdot \underset{\sim}{\mu}, \quad \mu \in \sim_{H^{1 / 2}}\left(\Gamma_{2}\right) .
\end{aligned}
$$


Note that $\underset{\sim}{\lambda}=\left.\underline{u}\right|_{\Gamma_{2}}$ and (4.7) is a weak statement of the traction boundary condition (4.3).

This formulation may be discretized in the usual way: the approximate solution is determined as a critical point of $\overline{\mathscr{L}}$ over a finite dimensional subspace $\underset{\approx}{S_{h}} \times V_{h} \times A_{h}$ of $\underset{\tilde{H}}{H}(\mathrm{div}, \Omega) \times{\underset{\sim}{L}}^{2}(\Omega) \times{\underset{\sim}{H}}^{1 / 2}\left(\Gamma_{2}\right)$. For ${\underset{\sim}{S}}_{h}$ and $\underline{V}_{h}$ we choose the same spaces as for the Dirichlet problem. In the next section we present a way to choose $A_{h}$ so that the finite dimensional problem admits a unique solution and derive error estimates for the three variables. First we discuss an approach to the computation of the discrete solution. We wish to compute $\left(\underset{\approx}{\rho_{h}}, \underline{\sim}_{h},{\underset{\beth}{h}}_{h}\right) \in{\underset{z}{h}}_{h}$ $\times V_{h} \times \mathbb{A}_{h}$ satisfying

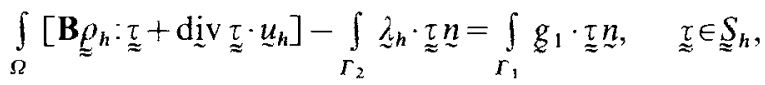

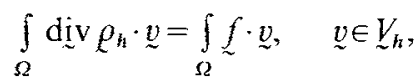

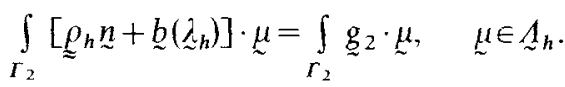

A direct solution of (4.8)-(4.10) involves a relatively large number of unknowns, so we indicate an alternative formulation in which only $\mathrm{z}_{h}$ is solved for directly.

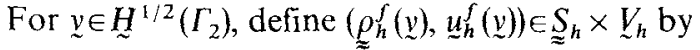

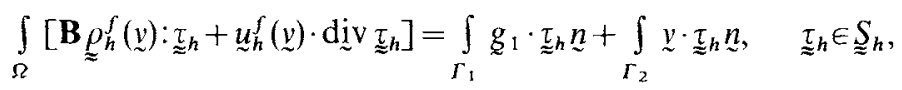

$$
\begin{aligned}
& \int_{\Omega} \operatorname{div} \underset{\sim}{\underbrace{f}_{h}}(v) \cdot v_{h}=\int_{\Omega} \underset{\sim}{f} \cdot \underline{v}_{h}, \quad \underline{v}_{h} \in{\underset{\sim}{h}}_{h} .
\end{aligned}
$$

Clearly (4.8) and (4.9) may be written equivalently as

and (4.10) becomes

$$
\left(\underline{\rho}_{h}, \underline{u}_{h}\right)=\left(\rho_{\tilde{z}}^{f}\left(\lambda_{h}\right), \underline{\sim}_{h}^{f}\left(\lambda_{h}\right)\right),
$$

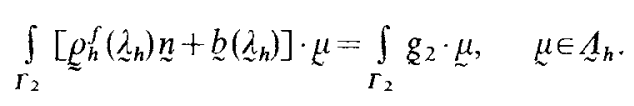

Via choice of a basis for $A_{h},(4.13)$ is reduced to a system of linear equations for the coefficients of $\lambda_{h}$. For an appropriate choice of elements this system is positive definite, as will be shown in the next section. In order to solve the system efficiently, one could employ a preconditioned conjugate-gradient iteration. The major cost of the iteration will be the repeated evaluation of the operator on the left hand side of (4.13), which in turn consists mainly of the solution of a displacement problem in order to evaluate $\underline{\sim}_{h}^{f}(\mu)$ for given $\mu$. Note that for all these displacement problems the operator is the same, and only the load is varied. To construct a preconditioner for this iteration, one can follow the ideas in Bramble [7]. Since the description of these results is fairly complicated, we do not include them here. 


\section{Stability for the Mixed Boundary Value Problem}

To discretize our formulation (4.5)-(4.7) of the mixed boundary value problem we must select subspaces $\underset{\approx}{S_{h}}, V_{h}$, and ${\underset{\sim}{h}}_{h}$. We shall suppose that $\underset{\approx}{S_{h}}$ and ${\underset{\sim}{h}}_{h}$ are chosen to be spaces which are effective for the Dirichlet problem in the sense that (3.13) holds, and determine $A_{h}$ so that the discrete problem (4.8)-(4.10) admits a unique solution which satisfies the quasioptimal estimate (cf. (3.14))

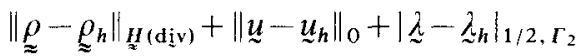

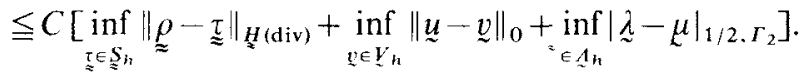

As is well-known, a necessary and sufficient condition for this, is that the method be stable in the sense that the bilinear form

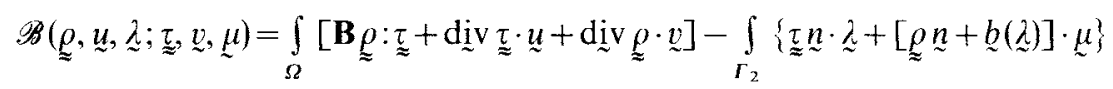

defined on $\left(\underset{\widetilde{S}}{S_{h}} \times \underline{V}_{h} \times{\underset{\sim}{h}}_{h}\right)^{2}$ satisfy the inf-sup condition [5]. We now give a simple sufficient criterion for stability. To state it we first define for each $\underset{\sim}{\underline{H} H_{\sim}}{ }^{1 / 2}\left(\Gamma_{2}\right)$ the pair $\left(\rho_{h}(y), u_{h}(y)\right) \in{\underset{\tau}{2}}_{h} \times \underline{V}_{h}$ as the solution of the discrete Dirichlet problem (4.11), (4.12) with $f$ and $g_{1}$ replaced by 0 .

Lemma 5.1. Assume that $\underset{\approx}{S_{h}}$ and $V_{h}$ satisfy (3.13) and that $\Lambda_{h}$ is chosen so that

$$
\int_{\Gamma_{2}}\left[\rho_{\sim}(\underline{\mu}) \underset{\sim}{n}+\underset{\sim}{b}(\mu)\right] \cdot \underline{\sim} \geqq \gamma|\underline{\sim}|_{1 / 2, \Gamma_{2}}^{2} \quad \text { for all } \underline{\sim} \in \mathcal{A}_{h} .
$$

Then (4.8) (4.10) defines a stable Galerkin method for the mixed boundary value problem.

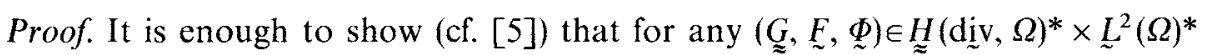
$\times H^{1 / 2}\left(\Gamma_{2}\right)^{*}$, the discrete variational problem:

Find $\left(\rho_{h}, \underline{u}_{h}, \lambda_{h}\right) \in{\underset{\sim}{h}}_{h} \times \underline{V}_{h} \times \underline{A}_{h}$ such that

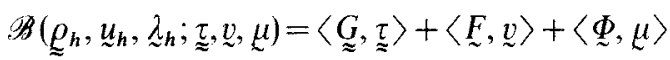

$$
\begin{aligned}
& \text { for all }(\underset{\tau}{\tau}, v, \mu) \in{\underset{\approx}{2}}_{h} \times V_{h} \times \Lambda_{h}
\end{aligned}
$$

has a unique solution, and that this solution satisfies

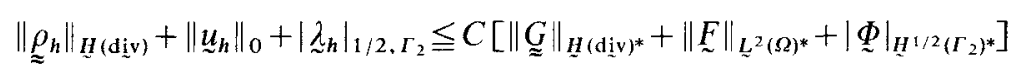

where $C$ is a constant independent of $h$.

Define $\left(\rho_{h}^{1}, u_{h}^{1}\right) \in \underset{\approx}{S_{h}} \times V_{h}$ by

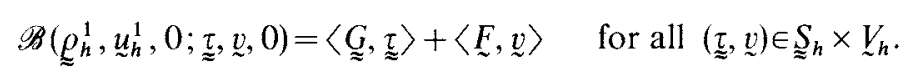

From the results of Sect. 3 there is a unique solution to this discrete Dirichlet problem and

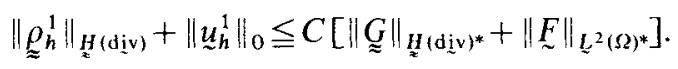


Upon subtracting (5.4) from (5.3) we see that it suffices to show that there is a unique $\left(\rho_{h}^{2}, u_{h}^{2}, \lambda_{h}\right) \in{\underset{\approx}{h}}_{h} \times V_{h} \times{\underset{\sim}{h}}_{h}$ such that

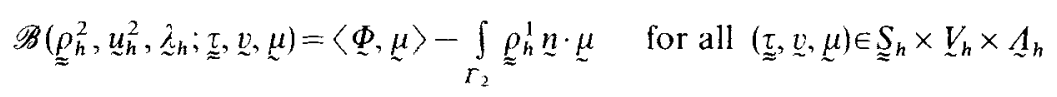

and that the estimate

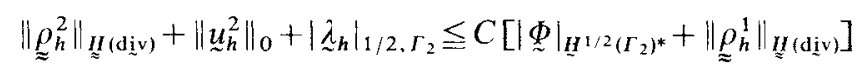

holds with $C$ independent of $h$. Clearly

$$
\left(\rho_{h}^{2}, \underline{u}_{h}^{2}\right)=\left(\rho_{h}\left(\lambda_{i}\right), \underline{\sim}_{h}\left(\lambda_{h}\right)\right)
$$

so we get using (5.2) and (5.5) that $\lambda_{h}$ is uniquely defined and

$$
\begin{aligned}
& \gamma\|\|_{h} \|_{1 / 2, \Gamma_{2}}^{2} \leqq \int_{\Gamma_{2}}\left[\underset{\sim}{\rho_{h}}\left(\lambda_{h}\right) \underset{\sim}{n}+\underset{\sim}{b}\left(\lambda_{h}\right)\right] \cdot \lambda_{h}
\end{aligned}
$$

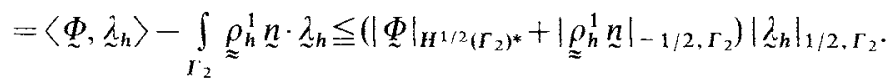

Thus

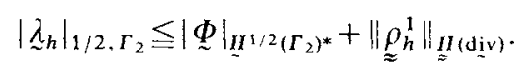

The proof is completed by invocation of (5.6).

We have attacked the traction boundary condition by modifying the Lagrangian for the Dirichlet problem through the addition of the variable $\lambda ;$; cf. (3.10) and (4.4). This is a technique commonly used when applying a Galerkin method to a problem with essential boundary conditions which cannot easily be imposed on the Galerkin subspace. For example, to solve a traction problem on a nonpolygonal domain using mixed finite elements based on the usual HellingerReissner principle, one likely possibility would be to use a multiplier method. For all such methods one must determine a suitable subspace in which to seek the new variable and a stability condition analogous to (5.2) must be verified implicitly or explicitly. In the particular case of scalar elliptic equations, there are a number of papers concerned with the choice of subspaces. See, e.g., [4, $7,8,17,18]$. Many of the approaches developed there could be adapted to our case. In the interest of brevity we will discuss only one, related to that of [4] and [7].

For definiteness we shall assume that $S_{h}$ and $V_{h}$ are chosen to be (Cartesian products) of the Raviart-Thomas-Nedelec elements of order $l$. Other subspaces with similar stability and approximation properties (e.g., those of Brezzi et al. [10]) could also be used with only small changes in the analysis. For the RaviartThomas-Nedelec elements, the approximation results

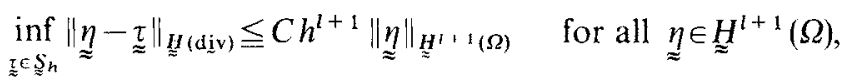

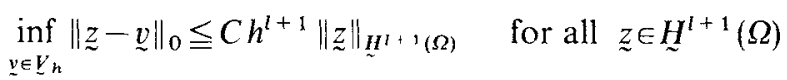


hold. In view of these results and in anticipation of the estimate (5.1), we assume that the space ${\underset{\sim}{h}}_{h}$ satisfies

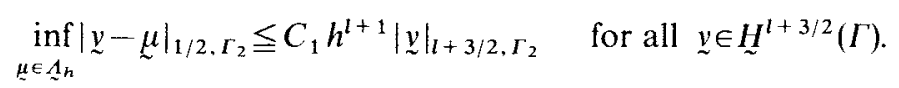

This is satisfied, e.g., if $A_{h}$ consists of continuous piecewise polynomials of degree at least $l$ with respect to a quasiuniform mesh of maximum element size $h$. The same choice guarantees the inclusion

$$
{\underset{\sim}{h}}_{h} \subset H^{1}\left(\Gamma_{2}\right)
$$

and the inverse inequality

$$
h^{1 / 2}|\underline{\sim}|_{1, \Gamma_{2}} \leqq C_{2}|\underline{\mu}|_{1 / 2, \Gamma_{2}} \quad \text { for all } \underset{\sim}{\mu} \in \underline{\sim}^{1}\left(\Gamma_{2}\right)
$$

for some constant $C_{2}$. The approach of [4] and [7] requires the inverse inequality (5.9) not merely for any $C_{2}$ but for a value sufficiently small. This can be achieved by taking $\Lambda_{h}$ to consist of piecewise polynomials subordinate to a mesh of elements which are not of size $h$, but rather of size $h / \varepsilon$. By selecting $\varepsilon$ sufficiently small, (5.9) can be achieved with any desired constant $C_{2}$. With this choice (5.8) of course still holds and so does (5.7) with $C_{1}$ replaced by $C_{1} / \varepsilon^{l+1}$.

To summarize: we assume that $\Lambda_{h} \subset H_{\sim}{ }^{1}\left(\Gamma_{2}\right)$ satisfies (5.9) for a value of $C_{2}$ to be specified and (5.7) for some value of $C_{1}$. Now we prove (5.2).

The verification of (5.2) relies on the fact that the analogous condition holds in the continuous limit. That is, there is a positive constant $\gamma_{0}>0$ such that

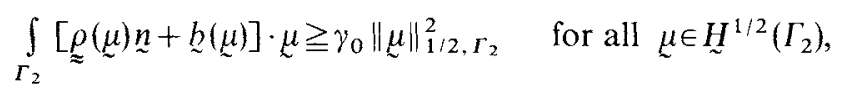

where $(\underset{\sim}{\rho(\mu)} \underset{\sim}{u}(\underset{\sim}{\mu}))$ is the solution of the displacement problem

$$
\begin{array}{cc}
\mathbf{B} \rho=\underset{\approx}{\underline{\approx}}(\underset{\sim}{u}) & \text { in } \Omega, \\
\operatorname{div} \underset{\sim}{\sigma}=0 & \text { in } \Omega, \\
\underset{\sim}{u}=0 & \text { on } \Gamma_{1}, \\
\underset{\sim}{u}=\underset{\sim}{\mu} & \text { on } \Gamma_{2} .
\end{array}
$$

To prove (5.10) let $\underset{\sim}{\sigma}(\mu)=\rho(\mu)-\beta \mathbf{D}(\operatorname{grad} u(\mu))$. Then the pair $(\underset{\sim}{\sigma}(\mu), \underline{\sim}(\mu))$ solves the homogeneous equations of elasticity (with compliance tensor A) and displacement boundary conditions (5.13), (5.14). From standard estimates $\int_{\Gamma_{2}} \underset{\sim}{\mu} \sigma(\underset{\sim}{g}) \underset{\sim}{n}$
$\geqq \gamma_{0}|\underline{\mu}|_{1 / 2, \Gamma_{2}}^{2}$, and (5.10) now follows from Lemma 4.1.

Next we bound the difference between the left hand sides of (5.10) and (5.2). By the Schwarz inequality and the trace inequality in $\underset{\approx}{\underset{\sim}{H}(\operatorname{div}}, \Omega)$,

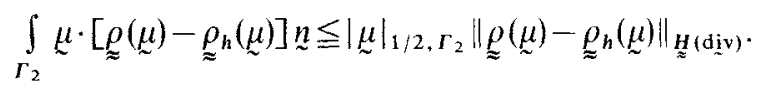


Using the fact that $\operatorname{div} \underset{\sim}{\rho} \underset{\sim}{\rho}(\mu)=\operatorname{div} \underset{\sim}{\rho_{h}}(\mu)=0$, standard estimates for the RaviartThomas-Nedelec elements ${ }^{1}$, and energy estimates for (5.11)-(5.14), we have

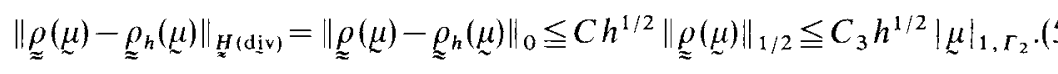

Combining (5.15), (5.16), and (5.9) gives

$$
\int_{\Gamma_{2}} \underset{\sim}{\mu} \cdot\left[\underset{\sim}{\rho}(\underset{\sim}{\mu})-\underset{\sim}{\rho_{h}}(\underset{\sim}{\mu})\right] \underset{\sim}{n} \leqq C_{2} C_{3}|\underset{\sim}{\mu}|_{1 / 2, r_{2}}^{2}
$$

Finally, choosing $C_{2}=\gamma_{0} /\left(2 C_{3}\right)$ and combining with (5.10) gives (5.2).

Using the quasioptimal error estimate (5.1) and the approximation properties of the subspaces $\underset{\approx}{S_{h}}, V_{h}$, and ${\underset{\sim}{h}}_{h}$, we get the error estimate

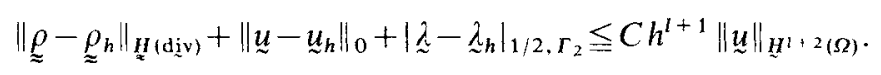

Using techniques developed for mixed and multiplier methods for scalar problems, it is possible to improve this estimate somewhat in the regularity required and also to derive estimates in other norms. Since the derivation of such results would be quite lengthy and only require minor modifications of the techniques for scalar problems, we do not include them here.

\section{Incompressible Elasticity}

We now consider the case of a homogeneous incompressible elastic material. Such a material with compliance tensor $\mathbf{A}$ is incompressible if $\mathbf{A} \delta=0$. In this case the displacement field satisfies $\operatorname{div} \underset{\sim}{u}=0$, as follows by taking the inner product of (3.1) with $\delta$. Since the compliance tensor is not positive definite on $\mathbb{R}_{s}$ for an incompressible material, the derivation of our formulation given in Sect. 3 does not apply. In this section we extend the results of Sect. 3 to the incompressible case. To do so we first note that the Dirichlet problem (3.1)(3.3) for an incompressible material admits a solution if and only if

$$
\int_{\Gamma} g \cdot \underset{n}{n}=0
$$

When (6.1) holds, the solution is uniquely specified by imposing the side condition

$$
\int_{\Omega} \operatorname{tr}(g)=0
$$

Our derivation is most easily understood for an isotropic material and so we consider that case first. For such a material the compliance tensor satisfies

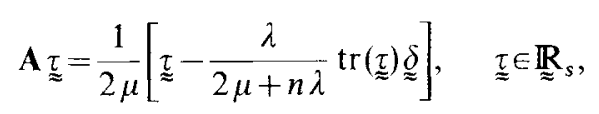

\footnotetext{
${ }^{1}$ Using the definitions of $\rho_{h}(\mu)$ and $\rho(\mu)$, it follows easily that $\left\|\rho(\mu) \cdots \rho_{h}(\mu)\right\|_{0} \leqq C\|\rho(\mu)\|_{0}$. Interpolating

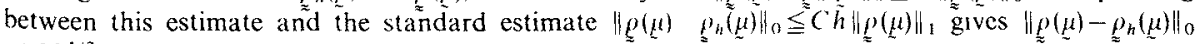
$\leqq C h^{1 / 2}\|\varrho(\mu)\|_{1 / 2}$
} 
where $\mu, \lambda>0$ are the Lamé constants and $n=2$ or 3 , the case $n=2$ corresponding to plane strain. For an incompressible isotropic material $\lambda=+\infty$, i.e.,

$$
\mathbf{A} \underset{\approx}{\tau}=\frac{1}{2 \mu}\left[\underset{\tau}{\tau}-\frac{1}{n} \operatorname{tr}(\underset{\approx}{\tau}) \stackrel{\delta}{\approx}\right]
$$

The least constant $c_{0}$ for which the upper bound in (2.1) holds is $c_{0}=1 /(2 \mu)$. Following the procedure of Sect. 3 at this point, we therefore choose $\beta=\mu$. For $\lambda<\infty$, we have

$$
\begin{array}{rlrl}
\mathbf{C} & =2 \mu \underset{\tau}{\tau}+\lambda \operatorname{tr}(\underset{\tau}{\tau}) \underset{\approx}{\delta}, & \underset{\approx}{\tau} \in \mathbb{\approx}_{s}, \\
(\mathbf{C}+\beta \mathbf{D}) \underset{\approx}{\tau}=\mu \underset{\approx}{\tau}+(\mu+\lambda) \operatorname{tr}(\underset{\tau}{\tau}) \delta, & & \underset{\approx}{\tau} \in \underset{\approx}{\mathbb{R}}, \\
\mathbf{B} \underset{\approx}{\tau}=\frac{1}{\mu}\left[\tau-\frac{\mu+\lambda}{(n+1) \mu+n \lambda} \operatorname{tr}(\tau) \delta\right], & \underset{\approx}{\tau} \underset{\approx}{\mathbb{R}} .
\end{array}
$$

The last equation is also valid for $\lambda=+\infty$, giving

$$
\mathbf{B} \underset{\approx}{\tau}=\frac{1}{\mu}\left[\tau-\frac{1}{n} \operatorname{tr}(\tau) \delta\right], \quad \stackrel{\tau}{\approx} \in \mathbb{R},
$$

in the incompressible case. The equation for the stress in terms of the pseudostress is

$$
\underset{\approx}{\sigma} \underset{\approx}{\rho}+\underset{\approx}{\rho^{t}}-\frac{2 \mu+\lambda}{(n+1) \mu+n \lambda} \operatorname{tr}(\underset{\approx}{\rho}) \underset{\approx}{\delta}
$$

for $\lambda<\infty$ or

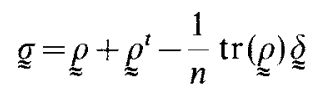

for $\lambda=+\infty$. Note that in both the compressible and the incompressible cases, $\operatorname{tr}(g)$ is a positive multiple of $\operatorname{tr}(\rho)$, so the side condition (6.2) holds if and only if

$$
\int_{\Omega} \operatorname{tr}(\underline{\rho})=0 .
$$

Let $\underset{\approx}{\hat{H}}(\operatorname{div}, \Omega)=\left\{\underset{\approx}{\tau} \underset{\approx}{H}(\operatorname{div}, \Omega): \int_{\Omega} \operatorname{tr}(\underset{\approx}{\tau})=0\right\}$. Then our formulation of isotropic incompressible elasticity seeks $(\rho, \underset{\sim}{u}) \in \underset{\tilde{H}}{\hat{H}}(\operatorname{div}, \Omega) \times \underline{\sim}^{2}(\Omega)$ as a saddle-point of the functional (3.10) with $\mathbf{B}$ defined by (6.3).

We shall now show that in fact there is a unique saddle-point. Recalling again Brezzi's theorem [9], we must verify two conditions. The second condition

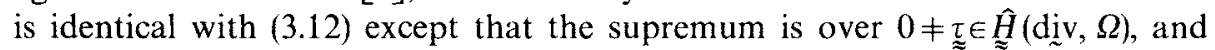
is again obvious. Condition (3.11) does not hold for any positive $\gamma$, since $\mathbf{B} \underset{\approx}{\approx}=0$. However Brezzi's theorem only requires that

$$
\int_{\Omega} \mathbf{B} \underset{\approx}{\tau}: \underset{\approx}{\tau} \geqq \gamma\|\tau\|_{\tilde{\tau}}^{2}, \quad \underset{\sim}{\tau} \in \underset{\approx}{Z},
$$

where

$$
\underset{\approx}{Z}=\{\underset{\approx}{\tau} \in \underset{\approx}{\hat{H}}(\operatorname{div}, \Omega): \operatorname{div} \underset{\sim}{\tau}=0\},
$$


and this is in fact so. For by a simple calculation

$$
\mathbf{B} \underset{\approx}{\tau}: \tau \frac{\tau}{\approx} \geqq \frac{1}{\beta}\left|\frac{\tau}{\approx}\right|^{2}, \quad \underset{\approx}{\tau} \in \mathbb{R}
$$

where $\underset{\approx}{\tau}=\underset{\approx}{\tau}-\frac{1}{n} \operatorname{tr}(\underset{\approx}{\tau}) \underset{\approx}{\delta}$ is the deviatoric of $\underset{\approx}{\tau}$ and it can be shown that

$$
\|\underset{\approx}{\|}\|_{0} \leqq C\|\underset{\approx}{\tau}\|_{0}, \quad \underset{\approx}{\tau} \underset{\approx}{Z},
$$

where $C$ depends only on $\Omega$. The bound (6.6) is well-known, at least when $\underset{\tilde{\mathbb{L}}}{\tau} \in \underset{\approx}{Z} \cap \underset{\approx}{\mathbb{R}}$, but the usual proof does not require symmetry of $\underset{\approx}{\tau}$. See, e.g., [3, Lemma 3.1].

In the compressible case we defined the pseudostress and displacement as a critical point of $\mathscr{L}$ over $\underset{\approx}{H}(\operatorname{div}, \Omega) \times L^{2}(\Omega)$ rather than $\underset{\tilde{H}}{\hat{H}}(\operatorname{div}, \Omega) \times L_{\tilde{\sigma}}^{2}(\Omega)$. Actually the latter choice of spaces may be used also for a compressible isotropic material when the Dirichlet data satisfies (6.1) since then

$$
\mathscr{L}\left(\underset{\tau}{\tau}-\left[\frac{1}{n} \int_{\Omega} \operatorname{tr}(\tau)\right] \underset{\approx}{\delta}, v\right) \leqq \mathscr{L}(\underset{\approx}{\tau}, v), \quad(\underset{\approx}{\tau}, v) \in \underset{\approx}{H}(\operatorname{div}, \Omega) \times L_{\sim}^{2}(\Omega) .
$$

The coercivity condition (6.5) holds uniformly for $\lambda \in[0, \infty]$. From this one can easily derive continuous dependence of $\underset{\approx}{\rho}, u$, and $\underset{\approx}{\sigma}$ on $\lambda$, up to and including $\lambda=\infty$.

A Galerkin discretization of our formulation with subspaces ${\underset{\tilde{W}}{h}}_{h} \subset \hat{H}$ (div, $\Omega$ ), $V_{h} \subset L_{\sim}^{2}(\Omega)$ is stable if the discrete Brezzi conditions hold, i.e., if the inequalities (3.13) and

$$
\int_{\Omega} \mathbf{B} \underset{\approx}{\tau}: \tau \geqq \gamma\|\tau\|_{\approx}^{2}, \quad \tau \in \underset{\approx}{\tau},
$$

hold for some $\gamma>0$. In (6.7),

$$
\underset{\approx}{Z_{h}}=\left\{\underset{\sim}{\tau} \in \underset{\approx}{S_{h}}: \int_{\Omega} \operatorname{div} \underset{\sim}{\tau} \cdot \underset{\sim}{v}=0 \text { for all } \underset{\sim}{v} \in V_{h}\right\} .
$$

Condition (6.7) holds if (3.16) does, since then $\underset{\approx}{Z_{h}} \subset \underset{\approx}{Z}$.

Finally we consider an anisotropic homogeneous incompressible material. For an incompressible material the identity $\varrho$ is an eigenvector of the compliance tensor with eigenvalue 0 . In order to extend our formulation to this case we first consider a material whose compliance tensor is positive definite on $\mathbb{R}_{\tilde{R}}$ and for which the identity is an eigenvector with eigenvalue $\varepsilon>0$. It is useful to introduce some notations for specific subspaces of $\underset{\approx}{\mathbb{R}}$ :

$$
\begin{aligned}
& {\underset{\approx}{\mathbb{R}}}_{a}=\left\{\underset{\approx}{\tau} \in \underset{\approx}{\mathbb{R}}: \tau_{\tilde{\tau}}^{t}=-\underset{\tau}{\tau}\right\}, \\
& \underset{\approx}{\mathbb{R}}=\{\underset{\approx}{\tau} \in \mathbb{\widetilde { R }}: \operatorname{tr}(\underset{\approx}{\tau})=0\}, \\
& \underset{\approx}{\mathbb{R}} s D=\underset{\approx}{\mathbb{R}} \cap \underset{\approx}{\mathbb{R}_{s}} \text {. }
\end{aligned}
$$




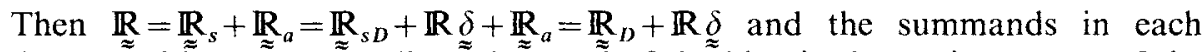
decomposition are mutually orthogonal. If the identity is an eigenvector of the compliance tensor, then the subspace $\underset{\approx}{\mathbb{R}_{s}}$ is mapped by the compliance tensor into itself and the restriction $\mathbf{A}_{0}=\left.\mathbf{A}\right|_{\mathbb{R}_{s D}}: \underset{\approx}{\mathbb{R}} \mathbb{z}_{s D} \rightarrow \underset{\tilde{\mathbf{A}}}{\mathbb{R}} s D$ is positive definite. In the incompressible case we shall also assume that $\tilde{\boldsymbol{A}_{0}}$ is positive definite, i.e, that 0 is a simple eigenvalue of $\left.\mathbf{A}\right|_{\mathbb{R}_{s}}$. The action of $\mathbf{A}$ may be described by

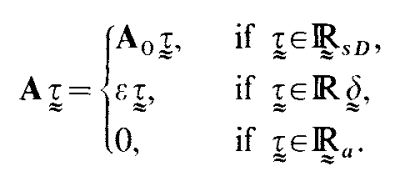

It follows that

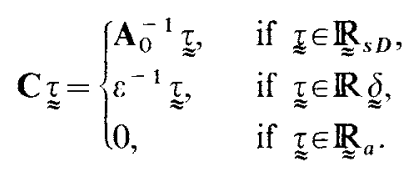

Now

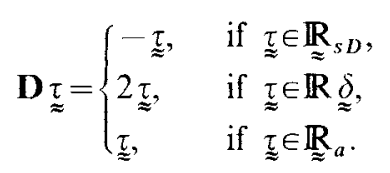

so

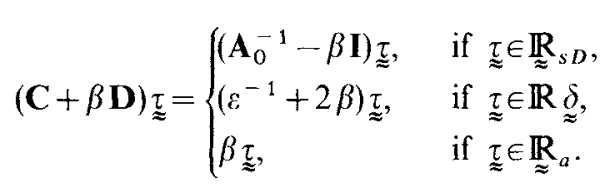

where, as before, $\beta$ is taken as $1 /\left(2 c_{0}\right)$ where $c_{0}$ is an upper bound for the eigenvalues of $\mathbf{A}$ and $\mathbf{I}$ is the identity. Thus

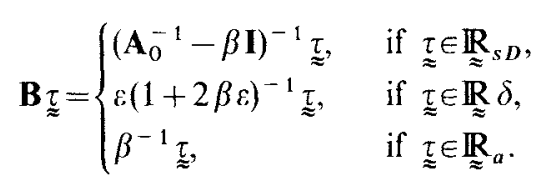

This last formula is also valid for $\varepsilon=0$, and we use it in the incompressible case. A unified formula for the tensor $\mathbf{B}$, valid for compressible and incompressible materials, is

$$
\mathbf{B} \equiv\left(\mathbf{A}^{\dagger}+\beta \mathbf{D}\right)^{\dagger},
$$

where the dagger denotes the Moore-Penrose inverse. This follows directly from the Moore-Penrose equations (see [6], p. 7).

Let us summarize these considerations. For an incompressible material, we assume that $\mathbf{A}_{0}=\left.\mathbf{A}\right|_{\mathbb{R}_{s D}}$ is positive definite, and let $\beta$ be half the reciprocal 
of its largest eigenvalue. Then $\mathbf{B}$ is well-defined by (6.8) with $\varepsilon=0$ or equivalently by $(6.9)$, and $(\rho, \underline{u})$ is defined as the saddle-point of $\mathscr{L}$ (given again by (3.10)) on $\hat{\tilde{H}}(\operatorname{div}, \Omega) \times \approx \underline{\sim}^{2}(\Omega)$. The saddle-point exists and is unique since $(6.5)$ and hence (6.4) holds. The pseudostress is related to the true stress by

$$
\underset{\approx}{\rho}=\underset{\approx}{\sigma}+\beta \mathbf{D}(\operatorname{grad} \underset{\approx}{u})
$$

The pseudostress satisfies the constitutive equation $\mathbf{B} \underset{\approx}{\rho}=\operatorname{grad} \underset{\approx}{u}$, so the true stress may be recovered without differentiating as

$$
\underset{\approx}{\approx} \rho-\beta \mathbf{D B} \rho
$$

\section{References}

1. Arnold, D.N., Brezzi, F.: Mixed and nonconforming finite element methods: implementation, postprocessing, and error estimates. RAIRO Math. Model. Numer. Anal. 19, 7-32 (1985)

2. Arnold, D.N., Brezzi, F., Douglas, Jr., J.: PEERS: A new mixed finite element for plane elasticity. Jap. J. Appl. Math. 1, 347-367 (1984)

3. Arnold, D.N., Douglas, Jr., J., Gupta, C.P.: A family of higher order mixed finite element methods for plane elasticity. Numer. Math. 45. 1-22 (1984)

4. Babuška, 1.: The finite element method with Lagrangian multipliers. Numer. Math. 20, 179-192 (1973)

5. Babuška, I., Aziz, A.K.: Survey lectures on the mathematical foundations of the finite element method. In: The Mathematical Foundations of the Finite Element Method with Applications to Partial Differential Equations (A.K. Aziz, ed.), pp. 5-359. New York: Academic Press 1972

6. Ben-Israel, A., Greville, T.N.E.: Generalized Inverses: theory and applications. New York: Wiley 1974

7. Bramble, J.H.: The lagrange multiplier method for Dirichlet's problem. Math. Comput. 37, 1-11 (1981)

8. Bramble, J.H., Falk, R.S. Two mixed finite element methods for the simply supported plate problem. RAIRO Anal. Numér. 17, 337-384 (1983)

9. Brezzi, F.: On the existence, uniqueness, and approximation of saddle-point problems arising from Lagrangian multipliers. RAIRO Anal. Numér. 2, 129-151 (1974)

10. Brezzi, F., Douglas, Jr., J., Marini, L.D.: Two families of mixed finite element methods for second order elliptic problems. Numer. Math. (to appear)

11. Douglas, Jr., J., Milner, F.A.: Interior and superconvergence estimate for mixed methods for second order problems. RAIRO Math. Model. Numer. Anal. 19, 397-428 (1985)

12. Douglas, Jr., J., Roberts, J.E.: Mixed finite element methods for second order elliptic problems. Mat. Apl. Comput. 1, 91-103 (1982)

13. Douglas, $f_{r}$, $f_{\text {, }}$ Roberts, J.E.: Global estimates for mixed methods for second order elliptic equations. Math. Comput. 44, 39-52 (1985)

14. Falk, R.S., Osborn, J.E.: Error estimates for mixed methods. RAIRO Anal. Numér. 14, 309-324 (1980)

15. Fortin, M.: An analysis of the convergence of mixed finite element methods. RAIRO Anal. Numér. 11, 341-354 (1977)

16. Nedelec, J.C.: Mixed finite elements in $\mathbb{R}^{3}$. Numer. Math. 35, 315-341 (1980)

17. Pitkäranta, J.: Boundary subspaces for the finite element method with Lagrange multipliers. Numer. Math. 33, 273-289 (1979)

18. Pitkäranta, J.: A conforming finite element method with Lagrange multipliers for the biharmonic problem. RAIRO Anal. Numér. 14, 309-324 (1980)

19. Raviart, P.A., Thomas, J.M.: A mixed finite element method for second order elliptic problems. 
In: Mathematical Aspects of the Finite Element Method. Lect. Notes Mathe. Vol. 606. Berlin, Heidelberg, New York: Springer 1977

20. Scholz, R.: $L_{x x}$-convergence of saddle-point approximation for second order problems. RAIRO Anal. Numér. 11, 209-216 (1977)

21. Scholz, R.: A remark on the rate of convergence for a mixed finite element method for second order problems. Numer. Funct. Anal. Optimization 4, 169-177 (1982)

22. Thomas, J.M.: Sur l'analyse numérique des méthods d'éléments finis hybrides et mixtes. Thèse, Université P. et M. Curie, Paris 1977

Received March 17, 1986/December 2. 1987 